\title{
A single molecule assay for measuring site specific DNA cleavage
}

Stefano Gambino ${ }^{a}$, Briana Mousley ${ }^{a}$, Lindsay Cathcart ${ }^{a}$, Janelle Winship ${ }^{a}$, Joseph J. Loparo $^{\mathrm{b}}$, Allen C. Price ${ }^{\mathrm{a},{ }^{*}}$

${ }^{a}$ Emmanuel College, Department of Chemistry and Physics, 400 The Fenway, Boston, MA 02115, United States

${ }^{\mathrm{b}}$ Harvard Medical School, Department of Biological Chemistry and Molecular Pharmacology, Boston, MA 02115, United States

* Corresponding author.

E-mail address: priceal@emmanuel.edu(A.C. Price)

Telephone: 1-617-975-9012 (A.C. Price)

\section{Abstract}

Sequence-specific DNA cleavage is a key step in a number of genomic transactions. Here, we report a single molecule technique that allows the simultaneous measurement of hundreds of DNAs, thus collecting significant statistics in a single experiment. Microbeads are tethered with single DNA molecules in a microfluidic channel. After the DNA cleavage reaction is initiated, the time of cleavage of each DNA is recorded using video microscopy. We demonstrate the utility of our method by measuring the cleavage kinetics of Ndel, a type II restriction endonuclease.

Keywords: DNA cleavage, DNA, Single molecule, Restriction endonuclease

Abbreviations:RE, restriction endonuclease; bp, base pair; FOV, field of view 
The cleavage of double stranded DNAat particular genomic loci occurs in a diverse group ofprocesses including V(D)J recombination, restriction modificationsystems, andgenome editingwithCRISPR/Cas9and TALENS. The most commonmethodused to assay DNA cleavage is gel electrophoresis. The limitedtime resolution and sensitivity of thistechnique has encouragedthe development of alternative methods including label free fluorescence[1], single molecule imaging[2], as well asoptical tweezers[3]. Single molecule techniquesoffer the ultimate in sensitivity, but often make acquiring statistically significant data difficult. Extending our previous work on flow stretching of DNA $[4,5,6]$, we have developed a multiplexing single molecule technique which measuresthe cleavage of hundreds of DNAs, providing statistically significant data in a single experiment.

Our approach is similar to others based on bead tethering that have been used to observeproteolysis at the single molecule level $[7,8]$. Magnetic microbeads are tethered with single DNAs in a microfluidic channel and are imaged using video microscopy (Fig. 1A). A constant flow rate through the microchannel, as well as a magnet positioned above the channel, apply small forces to the bead. Samples, containing enzymes or other effectors of DNA cleavage, can be introduced via flow into the channel. As its DNA is cleaved, each bead is carried up and out of the focal plane, disappearing from the video record at the moment of DNA cleavage. The video data can be analyzed to determine the "time to cleavage" of each individual DNA.We demonstrate our technique by measuring the kinetics of DNA cleavage by the type II restriction endonucleaseNdel. 
DNAs were linear 1000 basepair(bp) substratesconstructed using a polymerase chain reaction (PCR) with custom primers (IDT, Coralville, lowa) labeled on the $5^{\prime}$ end with either biotin or digoxigenin. M13mp18 plasmid (New England Biolabs, Ipswich, MA) was used as template DNA. The forward primer (digoxigenin labeled) was 5'-AAAAAAAAACGCGTTGGCCGA TTC-3' and the reverse primer (biotin labeled) was 5'-AGGGTGAGAAAGGCC-3'. A single Ndel cleavage site is located approximately $800 \mathrm{bp}$ from the digoxigenin labeled end of the DNA. The PCR products were purified using a Promega Wizard ${ }^{\circledR}$ PCR Clean-Up System (Promega, Madison, WI) following manufacturer instructions.

To construct the microfluidic channel, a3 in $\times 1$ in $\times 1 \mathrm{~mm}$ glass slide was predrilled to contain inlet and outlet ports for sample loading and removal. The inlet and outlet ports were connected to polyethylene tubing (PE60, Becton Dickenson \& Company, Waltham, MA) with epoxy. Inlet tubing was kept to no more than $8 \mathrm{~cm}$ in length to reducethe mixing time in the flow channel (see Supplementary Materials for discussion). Channels $2 \mathrm{~mm} \times 14 \mathrm{~mm}$ were cut in double sided tape (0.12 mm thick, Grace Bio-Labs, Bend, OR) and sandwiched between the glass slide and a $24 \mathrm{~mm} \times 60 \mathrm{~mm}$ No. 1 cover glass. To complete the assembly, the edges of the cover glass were sealed with epoxy.

To functionalize the flow channel for DNA tethering, $30 \mu \mathrm{L}$ of $20 \mu \mathrm{g} / \mathrm{mL}$ anti-digoxigenin antibody (Roche Diagnostics, Indianapolis, IN) in phosphate buffered salinewas loaded into the channel and incubated for $30 \mathrm{~min}$ at room temperature. After the incubation period, the channel was washed with $500 \mu \mathrm{L}$ of buffer A (20 mM Tris- $\mathrm{HCl}, 100 \mathrm{mM} \mathrm{NaCl}, 3 \mathrm{mg} / \mathrm{mL}$ BSA, $1 \mathrm{mg} / \mathrm{mL}$ Pluronic $\mathrm{F}-127,2 \mathrm{mM} \mathrm{MgCl}_{2}$ ). The flow cell was then mounted on an inverted 
microscope (Axiovert, Zeiss, Oberkochen, Germany)and connected to a syringe pump (Harvard

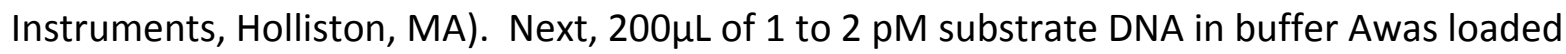
and incubated for 30 minutes. The channel was then washed with $500 \mu \mathrm{L}$ of buffer A before loading streptavidin coated micro beads ( $1 \mu \mathrm{m}$ diameter, MyOne Streptavidin beads, Life Technologies, Grand Island, NY) previously washed using a magnetic separator and prepared at a concentration of $3.2 \mu \mathrm{g} / \mathrm{mL}$ in buffer A. The magnetic microbeads were loaded at 2 to 10 $\mu \mathrm{L} /$ min until sufficient bead coverage was attained ( $\sim 15 \mathrm{~min})$. The flow cell was then washed with $500 \mu \mathrm{L}$ of buffer $\mathrm{A}$.

The site-specific endonuclease Ndel (New England Biolabs, Ipswich, MA) was diluted to the desired final concentration in buffer A. We tested the effect of flow rate on the measured cleavage rate and found little effect at rates $10 \mu \mathrm{L} / \mathrm{min}$ and below (see Supplementary Materials for discussion). Therefore, all enzyme solutions were continuously flowed through the microchannel at $10 \mu \mathrm{L} /$ min during data collection. Darkfield imaging was created by using an off-axis light source. Ring magnets (Magcraft, Vienna, VA) were held fixed approximately $8 \mathrm{~mm}$ above the sample cell to provide a weak magnetic force to reduce bead sticking. Video data was collected with an AxioCam HSM at 1 frame per second for approximately 30 min for each data set. All data was collected at $20^{\circ} \mathrm{C}$. To determine the number of uncleaved DNAs at each time point, the video data was reduced using automated particle counting (ImageJ,available from http://imagej.nih.gov/ii/). All fits to bead count data were determined using either linear regression or non-linear least squares (LoggerPro software, Vernier, Beaverton, OR). 
To reduce the chance of multiple DNAstethering asingle bead, we performed several tethering experiments with decreasing concentrations of DNA and noted the point at which the number of tethered beads decreasedrapidly asDNA concentration was reduced (typically around $1 \mathrm{pM})$. We performed all experiments with DNA concentrations near this level. Initial bead counts in our experiments varied from one hundred to a few hundred beads per field of view (FOV).

An example of our data is shown in Figure 1B, which shows the number of beads remaining in the FOV as a function of time. Due to the finite length of our inlet tube, the restriction endonuclease (RE) reaches the FOVat approximately 200s (time point labeled 2 in Fig. 1B). Between zero and 200s (region labeled 1 in Fig. 1B), the curve slopes downward due tonon-specificbead loss. The mechanism of this background bead loss is most likely due to the breakage ofthe digoxigenin-antidigoxigenin linkage or unbinding of the anti-digoxigenin from the cover glass to which it is non-specifically adsorbed. After 200s, the presence of Ndel causesthe bead loss rate to increase via enzyme catalyzed specific cleavage (region labeled 3 in Fig. 1B). In a typical data set, 20 to 30 beads would remain after completion of the reactiondue to beads sticking non-specifically to the cover glass. These remaining beads often exhibited a slow loss rate resulting in a slight negative slope to the measured baseline (region labeled 4 in Fig. 1B).

The background bead loss rate and the slight negative slope in the baseline visible in Fig. 1B introduce small errors into the data that can be removed by straightforward corrections. First, the baseline (labeled 4 in Fig. 1B) is fit to a linear function which is subtracted from the 
data. Next, the initial drop (labeled 1 in Fig. 1B) is fit to a single exponential decay which decays to zero. The background subtracted data is divided by thissingle exponential decay to produce the fully corrected data (see Supplementary Materials for a detailed example). The corrected data (Fig. 2) was well fit by an exponential decay of the form $P(t)=A e^{-k t}$, where $P(t)$ is the fraction of uncleaved DNAs at time $t, A$ is an amplitude, and $k$ is the rate of site specific DNA cleavage.

We demonstrated the application of our technique to measuring the apparent second order rate constant for site specific DNA cleavage. Figure 2 shows the dependency of the cleavagerate on enzyme concentration. The slope of thelinear relation (Fig. 2 inset)yields anapparent second-order rate constant of $(3.3 \pm 0.4) \times 10^{8} \mathrm{M}^{-1} \mathrm{~s}^{-1}$, close to diffusion controlledrates for enzymatic reactions [9]. Restriction endonucleases have been shown to locate their binding sites via facilitated diffusion which can involve one dimensional sliding along DNA[10,11,12]. Because we are measuring diffusion controlled rates, our results demonstrate that the method has the potential to measure the binding site"search time," a key parameter in the theory of facilitated diffusion [13].

Ourtechniquealso allows us to vary theforce on the micro bead and thus control the DNA tension. Many protein-DNA complexesshow significant bending [14], which raises interesting questions about the effect of tension on catalysis. In the case of restriction endonucleases, both tension and conformation have been shown to affect the cleavage rate $[3,15]$. In our experiments, the tension can be varied by changing the flow rate or the magnetic force. Increasing the flowmight affect the advection-diffusion of the enzyme to the 
surface and therefore complicatesthe interpretation of any change in measured reaction rates. Therefore, it makes more senseto vary the tension by changing the magnetic force on the microbead, which can be easily achieved by varying the magnet to sample distance.

The fact that our DNAs are attached to a solid support allows for varying the buffer conditions in situ during data collection. For example, we can pre-bind Ndel in the absence of $\mathrm{Mg}^{2+}$, an essential cofactor, without cleaving the DNA. Introducing $\mathrm{Mg}^{2+}$ into our flow channel then activates cleavage (data not shown), a result that is consistent with what is known for other REs $[2,16]$. Thisdemonstrates that thetechnique has the potential to study reaction intermediates that might occur after proteins have bound to their specific sites on DNA.

\section{Acknowledgements}

We would like to thank Wendy Gordon for supplying the initial DNAs used in this project. Support for this work also comes from a National Science Foundation CAREERAward MCB1148818 (to JJL) and the National Science Foundation RUI Award PHYS-1205814 (to ACP). 


\section{References}

[1] J. Deng, Y. Jin, G. Chen, L. Wang, Label-free fluorescent assay for real-time monitoring site-specific DNA cleavage by EcoRI endonuclease, Analyst 137 (2012) 1713-1717.

[2] W. Xu, S.J. Muller, Exploring both sequence detection and restriction endonuclease cleavage kinetics by recognition site via single-molecule microfluidic trapping, Lab on a Chip 11 (2011) 435-442.

[3] G.J. Gemmen, R. Millin, D.E. Smith, Tension-dependent DNA cleavage by restriction endonucleases: two-site enzymes are "switched off" at low force, Proceedings of the National Academy of Sciences 103 (2006) 11555-11560.

[4] B. Grafe, K. Williams, K. Burke, N. Tanner, A.M.v. Oijen, J. Loparo, A.C. Price, A single molecule DNA flow stretching microscope for undergraduates, American Journal of Physics 79 (2011).

[5] D. Song, B. Mousley, S. Gambino, E. Helou, J. Loparo, A.C. Price, Tethered particle motion with single DNA molecules, American Journal of Physics 83 (2015) 418-426.

[6] Allen C. Price, Kevin R. Pilkiewicz, Thomas G.W. Graham, D. Song, Joel D. Eaves, Joseph J. Loparo, DNA Motion Capture Reveals the Mechanical Properties of DNA at the Mesoscale, Biophys J 108 (2015) 2532-2540.

[7] W.R. Gordon, B. Zimmerman, L. He, L.J. Miles, J. Huang, K. Tiyanont, D.G. McArthur, J.C. Aster, N. Perrimon, J.J. Loparo, Mechanical allostery: evidence for a force requirement in the proteolytic activation of Notch, Developmental cell 33 (2015) 729-736.

[8] A.S. Adhikari, J. Chai, A.R. Dunn, Mechanical load induces a $\mathbf{1 0 0}$-fold increase in the rate of collagen proteolysis by MMP-1, Journal of the American Chemical Society 133 (2011) 16861689.

[9] A. Fersht, Structure and mechanism in protein science: a guide to enzyme catalysis and protein folding, Macmillan, 1999.

[10] D.C. Rau, N.Y. Sidorova, Diffusion of the restriction nuclease EcoRI along DNA, Journal of Molecular Biology 395 (2010) 408-416.

[11] I. Bonnet, A. Biebricher, P.-L. Porte, C. Loverdo, O. Bénichou, R. Voituriez, C. Escude, W. Wende, A. Pingoud, P. Desbiolles, Sliding and jumping of single EcoRV restriction enzymes on noncognate DNA, Nucleic Acids Res 36 (2008) 4118-4127.

[12] B. Terry, W. Jack, P. Modrich, Facilitated diffusion during catalysis by EcoRI endonuclease. Nonspecific interactions in EcoRI catalysis, Journal of Biological Chemistry 260 (1985) 1313013137.

[13] S.E. Halford, J.F. Marko, How do site-specific DNA-binding proteins find their targets?, Nucleic Acids Res 32 (2004) 3040-3052.

[14] R.E. Dickerson, DNA bending: the prevalence of kinkiness and the virtues of normality, Nucleic Acids Res 26 (1998) 1906-1926.

[15] B. van den Broek, M.C. Noom, G.J. Wuite, DNA-tension dependence of restriction enzyme activity reveals mechanochemical properties of the reaction pathway, Nucleic Acids Res 33 (2005) 2676-2684.

[16] A. Pingoud, M. Fuxreiter, V. Pingoud, W. Wende, Type II restriction endonucleases: structure and mechanism, Cellular and molecular life sciences 62 (2005) 685-707. 
Fig. 1.Single molecule DNA cleavage assay. (A) Experimental design. Paramagnetic microbeads functionalized with streptavidin are tethered to DNAs terminally labeledwith biotin.

Digoxigenin moieties atthe opposite end bind the surface functionalized with anti-digoxigenin. The magnet helps prevent non-specific sticking of beads. (B) Raw data. Plot shows number of uncleaved DNAs versus time. At time less than 200s (labeled 1) the decay is due to background bead loss (discussed in text). Enzyme reaches recorded FOV at 200s (labeled 2). The decay for time greater than 200s is increased due to enzyme catalyzed cleavage (labeled 3). The baseline is labeled 4.

Fig. 2. Cleavage rate dependence on Ndel concentration. Corrected data is shown: 70 pM (solid black), 43 pM (solid gray), 25 pM (dashed gray). Inset shows first order dependence of rate on concentration. Slope of fit yields a rate constant $(3.3 \pm 0.4) \times 10^{8} \mathrm{M}^{-1} \mathrm{~s}^{-1}$, consistent with a diffusion controlled reaction. Error bars represent standard errors in the mean. 
A
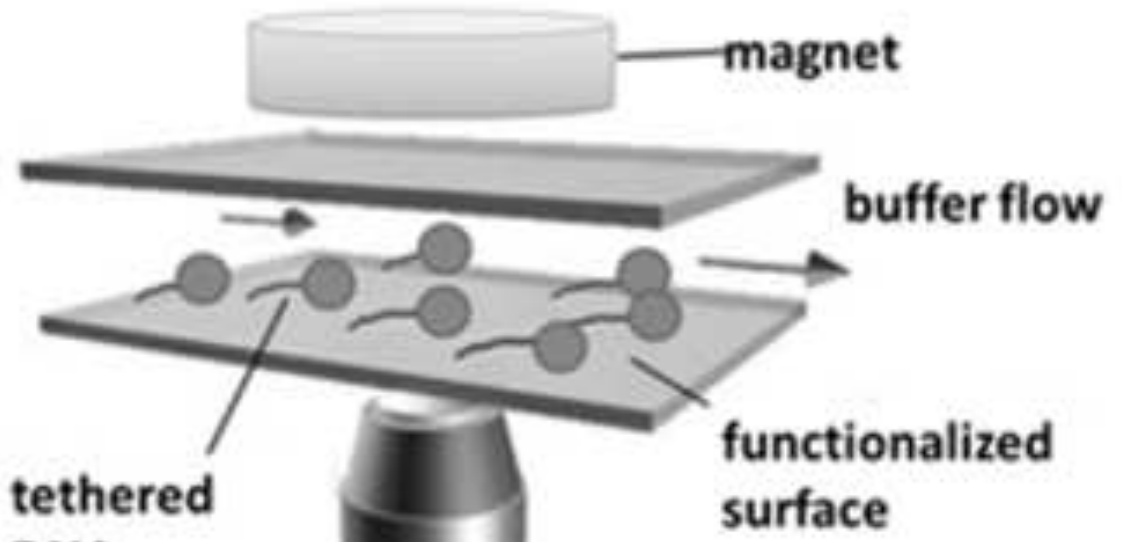

DNA
B

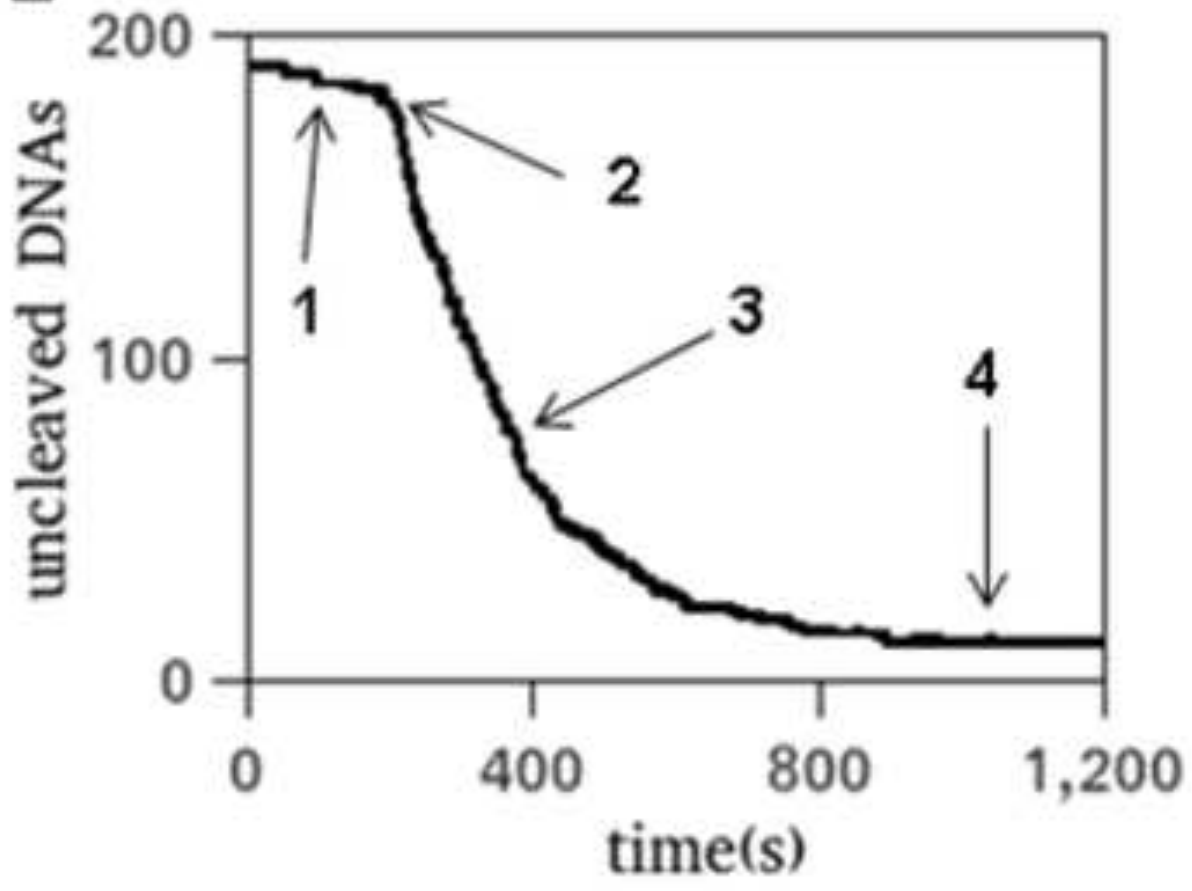




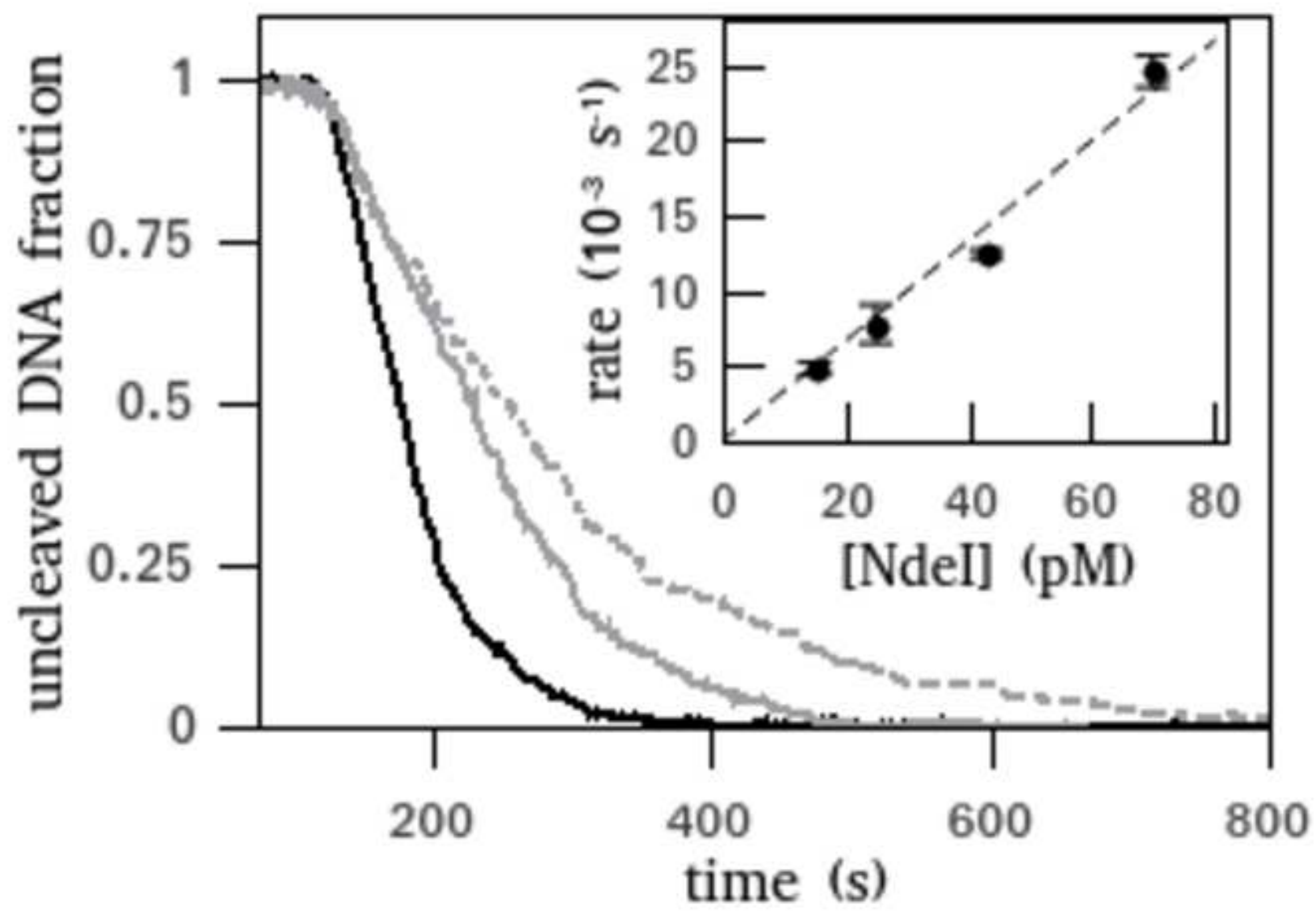

